\title{
Questes
}

\section{Construire la faute : la perfidie de l'empereur byzantin dans les sources latines de la première croisade}

\section{Annabelle Marin}

\section{(2) OpenEdition}

Journals

Édition électronique

URL : http://journals.openedition.org/questes/4222

DOI : $10.4000 /$ questes.4222

ISSN : 2109-9472

Éditeur

Les Amis de Questes

\section{Édition imprimée}

Date de publication : 30 octobre 2015

Pagination : 111-124

ISSN : 2102-7188

\section{Référence électronique}

Annabelle Marin, «Construire la faute : la perfidie de l'empereur byzantin dans les sources latines de la première croisade », Questes [En ligne], 30 | 2015, mis en ligne le 01 décembre 2015, consulté le 19 avril 2019. URL : http://journals.openedition.org/questes/4222 ; DOI : 10.4000/questes.4222 


\title{
Construire la faute : la perfidie de l'empereur byzantin dans les sources latines de la première croisade
}

\author{
Annabelle MARIN \\ Université Paris-Sorbonne
}

Timeo Danaos et dona ferentes. (Virgile, Énéide, Livre II)

Cette phrase de Virgile illustre tout à fait les relations complexes de méfiance et de fascination qui se sont instaurées entre les chefs croisés et l'empereur byzantin au cours de la première croisade. Les Occidentaux se sont méfiés des Grecs et, dans les chroniques, c'est en grande partie sur eux qu'est rejetée la faute, manquement moral qui consista à placer la conquête ou la reconquête de terres au centre des motivations de la croisade. Travailler sur les croisades implique d'analyser des chroniques diverses qui sont le reflet de personnalités et de circonstances particulières. L'étude porte non seulement sur le passé en tant que tel mais aussi sur ses représentations construites par les différents auteurs. Des points communs unissent tous ces récits, écrits dans le but de construire une mémoire, de prodiguer des exemples et de faire parfois le panégyrique d'un chef et d'un contingent. Néanmoins, deux types de récits se distinguent. Les récits originaux, rédigés par des témoins oculaires de la première croisade, tels que l'Anonyme des Gesta Francorum et aliorum Hierosolymitanorum, Foucher de Chartres et Raymond d'Aguilers qui restituent les actions de la première croisade, 
ainsi que les impressions immédiates des auteurs, ce qui n'est pas le cas des récits que l'on peut qualifier d' « indirects » tels ceux de Guillaume de Tyr, Robert le moine, Guibert de Nogent, Raoul de Caen et Albert d'Aix ${ }^{1}$. L'étude comparée du traitement des figures de l'empereur byzantin, Alexis I ${ }^{\mathrm{er}}$ Comnène, et de Bohémond d'Antioche, dans ces chroniques, donne à voir un exemple de construction littéraire de la faute.

Alexis Comnène, neveu de l'empereur Isaac Comnène et fils d'Anne Dalassène, avait commencé comme beaucoup d'autres jeunes nobles byzantins une carrière de soldat au service de l'empereur. En 1081, au moment où l'empire byzantin était dans une situation difficile, avec l'établissement des Turcs en Asie mineure et le danger normand qui se faisait de plus en plus menaçant à l'ouest, il s'empara du trône et réussit à écarter le danger extérieur et à pacifier la situation intérieure de l'Empire ${ }^{2}$. Bohémond d'Antioche ${ }^{3}$, prince de Tarente et fils de Robert Guiscard, jouait au même moment un rôle important dans la campagne menée par son père contre les territoires byzantins. Il ne s'agit pas de réhabiliter l'empereur, ni de réécrire l'Histoire mais de voir, en comparant le traitement qui lui est accordé dans les chroniques à celui accordé à Bohémond d'Antioche, comment sa perfidie a été construite dans les textes. Cette perfidie peut se définir comme un vice, un état, un trait de caractère. Dans les textes, Alexis Comnène n'est pas seulement fauteur de manière conjoncturelle, il est perfidus, c'est-à-dire trompeur, mauvais, déloyal et sournois, ce dont témoignent les nombreuses actions qu'il accomplit à l'égard des croisés et qui constituent des fautes morales.

1 Jean Flori, Chroniqueurs et propagandistes. Introduction critique aux sources de la Première croisade, Paris, Librairie Droz, 2010.

2 Élisabeth Malamut, Alexis $I^{\text {er }}$ Comnène, Paris, Ellipses, 2007. Le récit d'Anne Comnène constitue une source fondamentale pour le règne d'Alexis Comnène : Anne Comnène, Alexiade, éd. et trad. Bernard Leib, Paris, Les Belles Lettres, coll. « Collection Byzantine », 2006.

3 Jean Flori, Bohémond d'Antioche, chevalier d'aventure, Paris, Payot \& Rivages, coll. «Biographies Payot», 2007. 
Il va à l'encontre de la parole donnée lors de l'entrevue à Constantinople : per-fides. Ainsi, analyser dans le détail l'image de l'empereur byzantin, en la comparant à celle des chefs de la croisade et en la confrontant aux faits, permet aussi d'en saisir le caractère factice, entretenu par l'antagonisme qui opposa au Moyen Âge les Latins et les Grecs. Pourquoi Alexis Comnène est-il perfide, là où Bohémond d'Antioche n'est qu'habile politique ? Comment les chroniques construisent-elles la faute de l'empereur? Dans un subtil jeu de miroirs, les chroniqueurs ne se contentent pas de relater l'histoire mais construisent tout un univers mental en délivrant des leçons de morale. Édifiant et instruisant à la fois, ces récits sont bien des « arts de l'action ${ }^{4} »$.

\section{La construction de la perfidie de l'empereur}

Dès la mise en mouvement des troupes, Alexis Comnène adopta une attitude équivoque à l'égard des croisés, ce qui façonna son image d'empereur perfide auprès des Occidentaux et ce dès les premières années. Les rumeurs dont Raymond d'Aguilers se fit l'écho pendant la traversée de l'Esclavonie se vérifient: «on recevait des messages pacifiques de l'empereur, et en même temps nous étions entourés de toutes parts d'ennemis que nous suscitaient ses artifices ${ }^{5} »$. L'imposition des serments à Constantinople ainsi que les agissements de l'empereur lors du siège de Nicée font de ce dernier un être fourbe. On assiste à Constantinople et à Nicée à la genèse de l'image du perfide empereur, c'est-à-dire celui qui agit mal, celui qui trahit et qui faute. Avant de s'arrêter sur les mots et la manière de relater les actions de l'empereur, il faut évoquer les faits historiques.

4 Élisabeth Crouzet-Pavan, Le Mystère des rois de Jérusalem : 1099-1187, Paris, Albin Michel, coll. «Bibliothèque Albin Michel de l'histoire », 2013.

5 Raymond d'Aguilers, Histoire des Francs qui prirent Jérusalem, Paris, Les Perséides, 2006, p. 13. 


\section{Les faits}

Les croisés qui s'ébranlèrent en 1096, empruntèrent des itinéraires différents, mais ils se dirigeaient tous vers Constantinople, lieu de ralliement évident sur leur route. Selon Jean Flori, 30000 chevaliers se pressèrent sous les remparts de la ville ${ }^{6}$.

Alexis Comnène, étranger à l'idéal occidental de la croisade, était alors convaincu que le vrai dessein des seigneurs francs était de s'emparer de Constantinople. Ainsi une incompréhension s'instaura-t-elle entre les chefs et l'empereur, exacerbée par les préjugés anti-grecs déjà vifs à l'époque $^{7}$ mais aussi par le fait que certains négociateurs étaient des ennemis déclarés de l'Empire, tel Bohémond d'Antioche. Au terme de négociations, Alexis parvint à recueillir les engagements des plus grands chefs de la croisade, à l'exception de Raymond de Toulouse et de Tancrède. Quelle était la nature de ces engagements ? Concernant les liens juridiques qui unissaient les chefs croisés à l'empereur, les recherches de François-Louis Ganshof ${ }^{8}$ sont précieuses. L'analyse lexicologique n'est pas très pertinente car les termes employés par les chroniqueurs sont fluctuants et bien souvent très approximatifs. On relève en majorité les termes de foedus et juramentum, désignant des accords

6 Jean Flori, «Un problème de méthodologie. La valeur des nombres chez les chroniqueurs du Moyen Âge. À propos des effectifs de la Première Croisade », Croisade et Chevalerie, XI ${ }^{e}-X I I^{e}$ siècles, 1998, p. 332.

7 Voir Michel Balard, «Byzance vue de l'Occident », dans Dictionnaire raisonné de l'Occident médiéval, dir. Jacques Le Goff et Jean-Claude Schmitt, Poitiers, 1999, p. 126-135 et Michel Carrier, «Pour en finir avec les Gesta Francorum: une réflexion historiographique sur l'état des rapports entre Grecs et Latins au début du XII siècle et sur l'apport nouveau d'Albert d'Aix », Crusades, 7, 2008.

8 François-Louis Ganshof, Recherches sur le lien juridique qui unissait les chefs de la première croisade à l'empereur byzantin, Genève, Comité des mélanges P. E. Martin, 1961. On peut aussi se référer à Ralph-Johannes Lilie, Byzantium and the Crusader States, 1096-1204. Studies in the Relations of the Byzantine Empire with the Crusader States in Syria and Palestine [1981], trad. angl. James C. Morris et Jean. E. Ridings, Oxford, Clarendon Press, 1993, et John L. La Monte, « To what Extent was the Byzantine Empire the Suzerain of the Latin Crusading States? », Byzantion, 7, 1932, p. 253-264. 
officiels et signés dans la pure tradition occidentale, mais on peut cependant penser que l'ambiguïté et l'incompréhension s'installent. Si Alexis Comnène espérait une aide militaire de l'Occident et considérait ces chefs un peu comme des mercenaires ${ }^{9}$, les croisés firent preuve de discernement politique en redoutant de se placer dans la vassalité de l'empereur. Ils savaient pourtant qu'ils ne pouvaient continuer l'expédition «sans le conseil et l'aide de l'empereur»selon Foucher de Chartres ${ }^{10}$. En outre, Alexis adopta certains chefs de la croisade et offrit des otages à Godefroi de Bouillon, créant un lien supplémentaire avec les croisés. En effet, l'adoption est un rituel traditionnel dans le cérémonial de l'Empire, pouvant être appréhendé comme le pendant grec de l'engagement vassalique. Ainsi, à l'expression de «choc des croisades $^{11} »$, il faut plutôt préférer celle de rencontre manquée ou d'incompréhension mutuelle. La phase diplomatique terminée, les parties semblaient satisfaites, mais, déjà, une ambiguïté sourdait car tout semblait flou et indécis quant aux différentes interprétations à accorder à ces serments. Cet épisode démontre qu'Alexis mena une politique habile pour se rallier les chefs. L'accord politique et stratégique entre les croisés et l'empereur semblait parfait et, au-delà des querelles sur la définition des droits respectifs et la répartition des conquêtes, l'unanimité semblait de mise sur les premières opérations.

9 René Grousset précise d'ailleurs dans Histoire des croisades et du royaume franc de Jérusalem, Paris, Perrin, 2006, t. 1, chap. «L'anarchie musulmane et la monarchie franque », p. 87: «Les croisés devaient se considérer comme les soldats de l'Empire, tout comme les centaines de mercenaires francs qui depuis le début des siècles guerroyaient là bas au profit de l'autokrator, et l'Empire à son tour devait leur accorder comme à ceux-ci aide, ravitaillement, solde et honneur. ».

10 Foucher de Chartres, RHC, Sections historiens occidentaux, t. 3, Paris, 1844, p. 332.

11 Le Monde byzantin, dir. Jean-Claude Cheney, Paris, PUF, coll. « Nouvelle Clio », 2006, t. 2, chap. «L'Empire byzantin : 641-1204 », p. 52. 
Les récits des faits de la conquête doivent être considérés non pas pour les batailles qu'ils retranscrivent, mais pour ce qu'ils révèlent des acteurs et pour ce qu'ils font des personnages. En cela, la prise de Nicée est une étape essentielle, à la fois dans la conquête mais aussi dans la perception et la construction de la perfidie de l'empereur. Après les négociations à Constantinople, les croisés étaient au service de l'empereur et s'engageaient à lui rendre toutes les anciennes possessions byzantines qu'ils pourraient reconquérir sur les Turcs. L'empereur promettait quant à lui de les seconder, de prendre lui même la croix, et de les accompagner jusqu'au bout ou du moins d'adjoindre à leurs armées un contingent byzantin. Doit-on alors parler de la perfidie de l'empereur à Nicée ou de la prétendue perfidie de l'empereur? Pour Ferdinand Chalandon et René Grousset, Alexis Comnène n'a fait que mettre en application sa stratégie en exécutant les termes de l'accord passé à Constantinople, et ne serait donc pas perfide, car il n'irait pas à l'encontre du serment qu'il a prêté à Constantinople. Quand les croisés entrèrent en Asie mineure, la première ville à conquérir était Nicée, située en Bythinie et alors aux mains des Seldjoucides. Accompagnés par un contingent byzantin sous les ordres de Manuel Boutoumités, ils tinrent un siège de sept semaines mais furent laissés à l'écart des négociations et privés de l'assaut tant préparé contre les murailles de la ville. Au matin du 19 juin, il est aisé d'imaginer leur surprise en voyant les étendards de l'empereur flotter sur les tours de la ville qui s'était rendue dans la nuit ${ }^{12}$.

12 L'anonyme des Gesta exprime par exemple dans son récit la rancœur des guerriers de l'armée occidentale, privés des ressources escomptées du butin et du pillage : «Voyant qu'ils ne pourraient recevoir aucun secours de leurs armées, les Turcs envoyèrent une ambassade à l'empereur, s'engageant à lui rendre spontanément la ville s'il leur donnait les moyens d'en partir avec leurs femmes, leurs enfants et tous leurs biens. Alors l'empereur, possédé d'un vain et inique dessein, donna l'ordre de les laisser partir impunis sans avoir rien à craindre, et de les lui amener à Constantinople en toute confiance : il les épargnait avec ce zèle pour les avoir à sa disposition afin de nuire et faire obstacle aux francs »: Histoire anonyme de la 
Boutoumités fut nommé duc de la ville par l'empereur: les Byzantins accomplissaient leurs propres ambitions aux dépens des croisés. Le siège de Nicée n'altéra pas en apparence les bons rapports entre Alexis et les Latins et ne fut finalement qu'un jeu de dupes entre les différents acteurs qui repoussèrent tous deux l'affrontement direct et la rupture. Aucune confrontation directe, aucun incident ne survint. Mais les rapports se distendirent. Jean Flori évoque «une fissure ${ }^{13}$ » qui existait dès Constantinople et qui s'élargit à Nicée pour voir s'engouffrer les passions à Antioche.

Les faits étudiés et interprétés permettent à l'historien de dresser le portrait d'un empereur rusé et fin politique, menant à bien une stratégie de reconquête des terres sur les Turcs et dont les pratiques sont comparables à celles utilisées par les autres acteurs politiques du temps. L'image est celle d'un empereur fort et puissant qui arrive à s'imposer face aux barons, non celle d'un empereur perfide. Pourtant, du stratège au perfide, il n'y a que quelques mots, comme le démontre l'étude de la forme et de la construction des récits.

\section{Les mots des chroniqueurs}

L'analyse lexicologique des chroniques révèle que les récits de la première croisade sont hostiles à l'empereur dans leur ensemble. L'anonyme des Gesta surnomme l'empereur d'un bout à l'autre de son récit « le perfide empereur des Grecs ». Par ailleurs, ce même auteur ne manque pas de préciser que l'empereur a ressenti une très grande joie à la nouvelle du désastre de la croisade des pauvres et traduit le désarroi des

première croisade (Gesta Francorum et aliorum Hierosolymitanorum), trad. Louis Bréhier, Paris, Les Belles Lettres, coll. «Les Classiques de l'histoire de France au Moyen Âge », 1964, p. 41-42.

13 Jean Flori, La Première Croisade, l'Occident chrétien contre l'Islam. Aux origines des idéologies occidentales : 1095-1099, Bruxelles/Paris, Éditions Complexes, coll. «La mémoire des siècles », 2011, p. 75. 
chevaliers quand les chefs de la croisade prêtent serment de fidélité à l'empereur. Il reflète l'opinion de la plupart des croisés et ne perd pas une occasion de témoigner sa haine à l'égard d'Alexis qui est pour lui l'iniquus imperator, l'infelix imperator, l'imperator plenus vana et iniqua cogitatione $^{14}$. Si Foucher de Chartres semble plus indépendant et plus modéré que tous les autres, il est surtout très pragmatique et se plaint dès son arrivée à Constantinople du comportement d'Alexis Comnène, qui ne laissait pas les croisés séjourner à l'intérieur de la ville, leur causait des difficultés d'approvisionnement et ne leur permettait qu'une visite de la ville par petits groupes. Il se montre cependant réaliste et reconnaît que les traités passés entre l'empereur et les chefs croisés permettaient à ces derniers d'obtenir de l'argent et des chevaux. Louant l'habilité de l'empereur, son ton reste mesuré du fait qu'il servait à ce moment-là Etienne de Blois qui était tombé dès son arrivée à Constantinople sous le charme de l'empereur. Chez les autres chroniqueurs, les épithètes accolées au nom du basileus sont injurieuses. Le vocabulaire utilisé par Robert le Moine est lui aussi péjoratif: «artificieux empereur», « fourberie de l'empereur », « le rusé empereur », et même l'empereur « à l'esprit rempli de fraude » « renard d'empereur ». Selon ces chroniqueurs, l'empereur use en outre de flatteries (blanditia) et ses paroles sont mielleuses (mellita verborum). Il est intéressant de noter que les chroniqueurs reprennent ici l'héritage cicéronien et développent les caractéristiques propres au topos du graeculus, le «petit Grec». Enfin, tout au long du vaste récit de Guillaume, archevêque de Tyr, Alexis est qualifié de «la plus mauvaise bête féroce et l'homme le plus scélérat qui 
existe» ou de «prince impie qui commande aux Grecs». L'auteur évoque «la méchanceté », « la fraude » et «l'artifice » de l'empereur ${ }^{15}$.

Il faut aussi mettre en relief certains éléments. Les chroniques laissent en effet entrevoir une différence de perception entre l'empereur et la personne d'Alexis Comnène : ce n'est pas l'empereur en majesté, le continuateur de l'empire romain qui est incriminé mais bien la personne d'Alexis Comnène, fourbe et méchant. Les croisés sont fascinés par le décorum en place mais ils sont déçus par les actions et l'attitude de l'empereur. Ils détestent le grec Alexis Comnène, jeune général issu d'une famille aristocratique qui s'empare du pouvoir par un coup d'État au moment où l'empire byzantin, héritier de l'empire romain, est sur le point de sombrer. Il semble difficile pour un auteur occidental de cautionner et même de comprendre la pratique de l'usurpation, et il est passionnant de voir dans les textes l'apparition de topoï ${ }^{16}$. Illégitime, Alexis est de plus fourbe et méchant, lâche parce qu'il fuit le combat, rusé et indéchiffrable parce que grec, et Guillaume de Tyr ne manque pas de relever dans son récit les défauts des Grecs, notamment leur mollesse ${ }^{17}$.

15 L'empereur se définissant en permanence par sa «ruse», ses «artifices », son « double jeu », on peut se demander si les chroniqueurs, qui connaissaient l'Iliade, ne font pas d'Alexis Comnène un nouvel Ulysse, opposé à ces nouveaux Troyens que sont les croisés.

16 «L'Empire des Grecs était gouverné à cette époque par un homme méchant et plein de fourberie, nommé Alexis, et surnommé Comnène [...] Son rang le plaçait immédiatement après l'empereur; mais, se révoltant méchamment contre son maître et son bienfaiteur, il avait détrôné l'empereur et usurpé sa place cinq ou six ans avant l'arrivée des peuples d'Occident, et il osait se maintenir sur le trône après l'avoir occupé de vive force »: Guillaume de Tyr, RHC, op. cit., t. 1, livre II, ch. 5, p. 79.

17 «Il était facile de reconnaître quelles étaient alors la misère des Grecs et la faiblesse de leur empire, d'après l'état de ses contrées, où avaient été autrefois des provinces riches et fertiles, dans lesquelles on trouvait toutes les douceurs et tous les agréments de la vie. Lorsque l'empire de Constantinople, après l'extinction des princes latins, fut tombé, en punition de ses péchés au pouvoir du premier Nicéphore, les nations barbares, comptant sur la faiblesse des Grecs, se précipitèrent dans leurs provinces, et traitèrent au gré de leurs caprices les habitants de ce pays. » : Guillaume de Tyr, RHC, op. cit., t. 1, livre IX, ch. 5, p. 371. 
Ils sont vus comme indignes de leur empire. Finalement, l'empire byzantin, tel que le découvrent les croisés, est à l'image des Grecs, faible et malheureux, dans une situation difficile. L'analyse des mots des chroniqueurs montre donc la construction de l'image d'un empereur perfide là où les faits ne traduisent qu'une attitude équivoque de la part de l'empereur. Après avoir vu comment les chroniqueurs, peut-être à la suite d'une incompréhension plus que d'un choc, construisaient cette image d'empereur perfide, image qui s'appuie sur des faits dont la portée et l'interprétation sont discutables mais aussi sur des mots, il faut voir quels en étaient les buts.

\section{Le traitement du conflit entre Bohémond d'Antioche et Alexis}

Comnène dans les chroniques : reflet du divorce entre Orient et Occident ?

Dans les chroniques, les chefs apparaissent bien comme des héros. Cependant, confrontés à l'image de l'empereur byzantin, les héros ne sont pas invincibles, mais bien faillibles. Leurs fautes, erreurs et échecs sont minimisés et finalement peut-être excusables là où la perfidie de l'empereur n'est que condamnable. Alexis Comnène se présente dans les chroniques comme un empereur aux multiples visages, miroir inversé de tous les chefs, car le rejet et l'opposition constituent des ressorts importants de ces chroniques. Si, au début de la conquête, les barons semblent ne faire qu'un contre Comnène, dans les récits, peu à peu, chaque chroniqueur construit un idéal, un modèle, un mode de pensée et un mode d'action qui se présente à un premier niveau de lecture comme le contrepoint de la figure de l'empereur. Dans les récits, les chefs sont bien souvent courageux quand l'empereur fuit le combat. Le travail de l'historien permet de déconstruire les jugements des chroniqueurs et les 
topoï littéraires qu'ils bâtissent. Dans chaque chronique, une rhétorique originale de l'altérité se met en place. Cette lecture n'est possible que par l'entrée des parties concernées dans un même système, entrée réalisée par les serments à Constantinople et les fissures de Nicée puis Antioche. Car dire l'Autre c'est le poser comme différent. Dès lors que la différence est dite ou retranscrite, elle devient significative, puisqu'elle est prise dans les systèmes de la langue et de l'écriture. Pour traduire cette différence, le chroniqueur dispose du procédé de l'inversion, où l'altérité se transcrit en « anti-même »: on n'oppose plus le chef croisé à un autre chef mais le chef croisé à son inverse. Cependant la transcription se révèle être inaboutie et imparfaite, et l'analyse du contexte et des faits démontre que l'Autre devient plus proche. Les actions des chefs et de l'empereur doivent plutôt être lues en termes de gradation et d'échelle de la faute et de l'erreur. Plus particulièrement, on peut opposer Alexis Comnène à Bohémond d'Antioche, son adversaire le plus acharné et le plus dangereux. Le chef normand et l'empereur ont tous deux la même intelligence politique. En ce qui concerne par exemple les motivations de Bohémond d'Antioche pour partir en croisade, Tudebode et l'auteur anonyme des Gesta dépeignent un chevalier pieux, véritable champion du christianisme qui aurait été brusquement saisi par le saint Esprit pour se métamorphoser en croisé modèle ${ }^{18}$. Geoffroy Malaterra et Anne Comnène, plus pragmatiques, évoquent plutôt un aventurier uniquement préoccupé de piller et de s'emparer des terres byzantines. Le départ précipité de Bohémond vient corroborer ces portraits et dévoile son véritable dessein, qui est avant tout politique. La ruse et la perfidie de

18 « Aussitôt incité par l'Esprit saint, Bohémond ordonna de découper un précieux manteau qu'il portait et en fit distribuer les morceaux découpés en croix. [...] De retour dans sa terre, le seigneur Bohémond se prépara avec zèle à prendre le chemin du Saint Sépulcre. »Histoire anonyme de la première croisade, op. cit., p. $18-19$. 
Bohémond d'Antioche semblent alors excusables. Pourtant, le revirement accompli par le chef normand à Antioche n'est-il pas digne de la perfidie de l'empereur ${ }^{19}$ ? Bohémond parvint en effet à ses fins à Antioche par ruse car il venait de pactiser avec un Arménien, gardien d'une tour de la ville et était assuré de rentrer dans la ville le premier. Après des tensions avec le comte de Toulouse, il finit par s'établir seigneur de la ville d'Antioche. En janvier 1099, la croisade repartit vers Jérusalem tandis que Bohémond resta à Antioche. Le fait que Bohémond ne reparte pas avec les autres croisés fonde les craintes de l'empereur et montre que le chef normand n'éprouvait en fait pas un grand désir de se rendre à Jérusalem. Alexis Comnène et Bohémond d'Antioche se ressemblent donc plus qu'ils ne s'opposent ${ }^{20}$ : ce sont des hommes politiques qui se sont avant tout servi de la croisade pour conquérir des terres. L'empereur était parvenu à récupérer une partie de ses terres en Asie tandis que le chef normand avait fini par conquérir Antioche. Si les actions de l'un sont condamnées et sévèrement retranscrites et jugées dans les récits, celles de l'autre sont cachées ou du moins minimisées et on assiste bien, en ce qui concerne l'empereur, à la construction littéraire d'une faute. L'affrontement entre les deux hommes après la prise d'Antioche est ouvert. Aux émissaires de l'empereur qui l'enjoignent de se retirer de la ville, Bohémond répond en accusant en retour le basileus d'avoir luimême provoqué la rupture du serment en ayant abandonné les croisés et en n'ayant pas agi comme un souverain. Par conséquent, dans la logique des sources occidentales, Bohémond d'Antioche ne trahit pas son serment

19 Bohémond, alors homme de confiance de l'empereur, se transforme en ennemi. René Grousset, Histoire des croisades, op. cit., précise : « Ne pouvant se servir de Byzance, il renonça à la servir. Désespérant de jamais obtenir Antioche des mains des Comnènes, il résolut de l'obtenir contre eux. »p.146.

20 Jonathan Shepard, «When Greek meets Greek: Alexius Comnenus and Bohemond in 1097-1098 », Byzantine and Modern Greek Studies, 12, 1988, p. 185-277. 
lorsqu'il saisit Antioche : Alexis ayant manqué à son devoir, il n'est plus seigneur, et donc il n'y a plus de serment.

Cependant, comprenant que la situation lui échappait et qu'Alexis Comnène était progressivement en train de l'emporter, Bohémond d'Antioche décida alors de confier la ville à Tancrède et de partir mener en Occident ce que René Grousset n'hésita pas à qualifier de «croisade anti-byzantine $^{21} \gg$. Déployant une véritable propagande ${ }^{22}$, il fit circuler l'idée de la perfidie d'Alexis, qui passait pour un traitre à la chrétienté, et l'on peut considérer que c'est au cours de cette période que se forgèrent en Occident les préjugés sur les Byzantins considérés comme perfides, efféminés et schismatiques.

Construire une image négative de l'empereur, insister sans cesse sur la faute morale que constituent ses trahisons servait les chroniqueurs. La perfidie ou plutôt les perfidies d'Alexis Comnène, tour à tour mauvais seigneur et mauvais chrétien ${ }^{23}$, per-fides, allant à l'encontre de la foi et de la fidélité, permettaient non seulement de glorifier les chefs mais aussi d'excuser leurs propres fautes, car les chroniqueurs ne semblent pas avoir été dupes du caractère faillible des hommes dont ils retranscrivaient les hauts faits mais aussi les fautes, les erreurs et les échecs. À une échelle plus large, on peut même se demander si ce n'était pas une nécessité pour eux d'incriminer l'empereur comme pour mieux justifier la fondation des États latins d'Orient. Ainsi, les récits de la première croisade sont bien

21 René Grousset, Histoire des croisades, op. cit., p. 456.

22 Jean Flori, «De l'anonyme normand à Tudebode et aux Gesta Francorum. L'impact de la propagande de Bohémond sur la critique textuelle des sources de la première croisade », Revue d'Histoire Ecclésiastique, 2007, vol. 102, N ${ }^{0}$ 3/4, p. 717-746.

23 Michel Carrier, L'Autre chrétien pendant les croisades. Les Byzantins vus par les chroniqueurs du monde latin (1096-1261), Editions universitaires européennes, Saarbrücken, 2012. 
des « arts de l'action et de la mémoire ${ }^{24} »$. Écrire l'histoire de la croisade, c'est agir, dans le présent et pour le futur. C'est à la fois atténuer la faute de l'un et condamner celle de l'autre à travers des procédés littéraires. L'analyse des chroniques des croisades ne permet pas seulement de penser les succès et les gloires mais aussi d'entrevoir une vision de l'échec et de l'erreur des hommes à travers une intrigue, celle de l'iter hierosolymitanum. La première croisade constitue en effet un autre temps fort dans les relations entre l'Orient et l'Occident après le schisme de 1054. A l'expression « choc de la croisade », il convient plutôt de préférer celle d' « incompréhension mutuelle », car les Occidentaux ont à l'époque une très mauvaise connaissance de l'Orient et des Orientaux et les chroniqueurs des croisades ne font pas exception. L'Orient tant musulman que grec apparaît dans les écrits comme un vaste espace saturé de représentations et de significations, un ensemble riche, complexe et indéchiffrable. La littérature de la croisade constitue un observatoire privilégié pour penser, comprendre, saisir la perception de l'échec, de l'erreur et de la faute au Moyen Âge, ce « revers de la médaille » évoqué en introduction du thème. Combien de sièges, de trahisons sont dépeints dans ces textes! Non seulement en ce qu'ils constituent de puissants ressorts dramatiques, mais aussi parce qu'ils divulguent des leçons, des exemples de morale. Bien loin des clichés opposant héros et méchants, bons et mauvais, les chroniqueurs médiévaux savent délivrer des leçons de vie et des enseignements tout en complexité en convoquant dans leurs écrits de multiples procédés littéraires.

24 Élisabeth Crouzet-Pavan, Le Mystère des rois de Jérusalem 1099-1187, op. cit. 